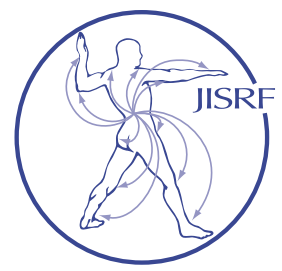

\title{
Addressing a Complex Proximal Femoral Deformity With Custom Cutting Guides Using 3D-Computer Design Software: A Case Report and 2-year Follow-Up
}

Hanson, $Z^{1}$; Davis, $D^{1}$; Robison, $\mathrm{J}^{1}$; Minter, $\mathrm{J}^{2}$

\section{Abstact}

We report a case of a 23 -year-old female with a history of congenital proximal femoral deformity and malunion of a prior proximal femoral corrective osteotomy, who presented seeking treatment for debilitating end-stage arthritis of her hip. Consideration for total hip arthroplasty (THA) for this patient was complicated by her young age and the complexity of her proximal femoral deformity. A 3-dimensional bone model of the patient's femur was created using digital reconstructive software based on preoperative CT-imaging and used to plan our corrective osteotomy and arthroplasty component specifications. Using the detailed characterization of the femoral morphology, custom cutting guides were designed to fit uniquely into the correct position and ensure a high degree of accuracy with our osteotomy cuts. This unique case highlights the use of 3D-modeling software and printing technology for detailed surgical planning and precise execution in patients with complex deformities or otherwise abnormal anatomy.

\section{Background}

Proximal femoral deformity (PFD) may refer to varus or valgus femoral neck-shaft angles, rotational malalignments, leg-length discrepancies or any combination. In adults, PFD may be caused by a number of congenital (e.g., fibrous dysplasia, coxa vara, developmental dysplasia) or acquired etiologies (e.g., infection, fracture malunion, prior proximal femoral osteotomy). [1,2] Regardless of etiology, PFD alters lower extremity mechanical alignment and creates abnormal joint surface stress, leading to accelerated joint surface wear and early arthritis [ $\underline{3}-7]$.

One of the most important treatment goals in these patients is early deformity correction with proximal femoral osteotomy (PFO) to restore mechanical alignment of the lower extremity, provide pain relief, improve gait and overall function. [] Even after early intervention, many of these patients go on to develop significant hip osteoarthritis at a relatively young age, in which case arthroplasty may be considered. Standard hip replacement techniques and prostheses are typically unsuitable for patients with significant PFD; abnormal femoral morphology may limit adequate component placement and the altered mechanical alignment can lead to accelerated component wear and increased rates of aseptic loosening. Performing adjunctive proximal femoral osteotomies in these patients can restore bony anatomy, simplifying the procedure and improving arthroplasty implant survival.

Proximal femoral deformity correction is technically

Keywords: Proximal Femoral Deformity, Patient-Specific Instrumentation; Proximal Femoral Osteotomy, $3 D$ printing osteotomy template, Three-Dimensional

Level of Evidence: $V$ 
challenging and surgical planning must consider osteotomies to address malalignment, often in multiple planes, as well as choice of hardware to facilitate the degree of surgical correction. [2-13] Even after thorough preparation it can be difficult to accurately replicate the degree of correction planned preoperatively. Advances in computer technologies has led to surgeons seeking support through navigation systems, digital planning tools, and more recently, $3 \mathrm{D}$ digital reconstruction and $3 \mathrm{D}$ printing techniques to more effectively plan and improve surgical precision to achieve more reliable outcomes.

\section{Case History}

A 23-year-old female presented to our clinic with the complaint of constant right-sided groin and thigh pain, which worsened with activity. Her symptoms had developed gradually and progressed in severity over several years. At the time of presentation, she reported difficulties activities of daily living including stair climbing and application of shoes and socks to the affected extremity, and a maximum ambulatory distance of a quarter mile. The patient's history was significant for severe right-sided femoral deformity due to a slipped capital femoral epiphysis (SCFE), for which she had undergone in-situ pinning and attempted deformity correction with a Southwick (subtrochanteric) osteotomy at an outside medical facility. Of note, the patient also had significant pelvic tilt and spinal deformity due to scoliosis. All retained femoral hardware had been removed years prior to our initial encounter.

On physical exam the patient's right hip exhibited significantly restricted range of motion with approximately 60 degrees of flexion, 20 degrees of abduction, 15 degrees of adduction, 15 degrees of external rotation and 0 degrees of internal rotation. On strength examination of the hip, the patient exhibited $4 / 5$ strength with hip abduction, adduction and hip flexion. Limb lengths were measured from umbilicus to medial malleolus and determined to have approximately $3 \mathrm{~cm}$ of discrepancy $(96 \mathrm{~cm}$ on the right and $99 \mathrm{~cm}$ on the left). She had a short limbed, antalgic, nonTrendelenburg gait. Interestingly, the patient exhibited hyper-mobility at her elbow, wrist and knee joints bilaterally.

Radiographs demonstrated a complex multi-angular deformity with severe osteoarthritis of the right hip. Evidence of this patient's original SCFE deformity is seen on plain films (Fig. 1), which demonstrate coxa vara and proximal femoral retroversion. An extension deformity has developed due to posteroinferior displacement of the femoral head in relation to the femoral neck. The metaphysis of the anterolateral neck has formed a CAM-type lesion
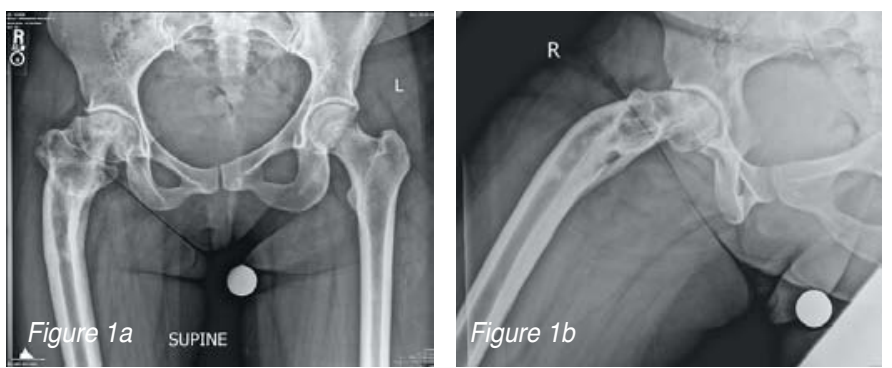

Figure 1. Preoperative radiographs showing (a) an anteroposterior view of the pelvis and (b) a cross-table lateral view of the patient's right hip. Radiographs show marked coxa varus and proximal femoral retroversion, with associated posteroinferior displacement of the femoral head in relation to the femoral neck and shaft consistent with history of SCFE.

due to abutment against the anterior acetabulum. Dense sclerotic cortical bone is noted with gross shortening of the extremity and associated pelvic tilt. The degree of femoral retroversion and anterior metaphyseal abutment here are consistent with the restrictions to hip flexion and internal rotation seen on clinical exam.

The patient presented after having delayed surgery as long as possible, though she was now at a point where her pain and functional limitations warranted intervention. While the degree of arthritis present warranted treatment with joint reconstruction, the complexity of her deformity prohibited conventional arthroplasty techniques; her abnormal canal morphology increased the risk for intraoperative complications (e.g., fracture), poor fit of standard components and, in combination with her altered joint mechanics, early implant failure.

To address these concerns, surgical treatment would involve deformity correction with proximal femoral osteotomies and subsequent total hip arthroplasty. Given the complexity and multi-plane nature of the deformity, 3-dimensional (3D) surgical planning using CustomLINK (Waldemar Link GmbH and Co.; Hamburg, Germany) was used to more accurately plan osteotomy cuts that provide the desired angular correction. Based on fine-cut CT images (coronal and sagittal reformations), digital reformatting software was used to generate a 3D-model of the right hemipelvis and femur (Fig. 2). A dedicated engineering team was available to assist with osteotomy planning, design custom-cutting guides and provide input on implant size and design.

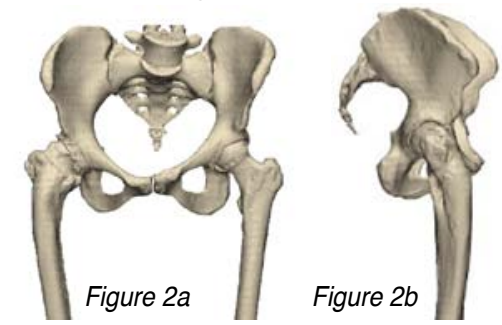

Figure 2. Anterior (a) and lateral $(b)$ views of the 3-dimensional reconstructed model based on fine-cut CT imaging of the pelvis and proximal femur. 
In collaboration with the engineering team, a single osteotomy was planned at a location which would provide optimal angular correction (Fig. 3). Planning a single oste-

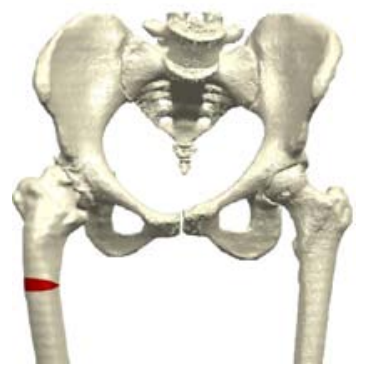

Figure $3 a$

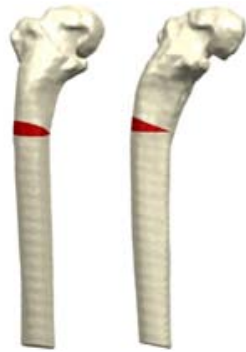

Figure $3 b$

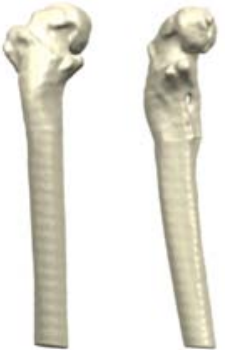

Figure $3 c$
Figure 3. Preoperative 3-dimensional planning for the proximal femoral corrective osteotomy shows the planned wedge osteotomy denoted in red on the (a) anterior view of the pelvis and (b) anterior and lateral views of the isolated femur. By digitally removing the osteotomy wedge, the final alignment based on the projected correction can be assessed in multiple planes (c).

otomy cut was ideal, provided it achieved sufficient deformity correction, because it allowed for a simplified, "one and done" design for a cutting jig. A custom cutting-guide based on this patient's unique bony topography was designed using 3D printing technology and used to help ensure the angular correction planned preoperatively was replicated during surgery (Fig. 4).

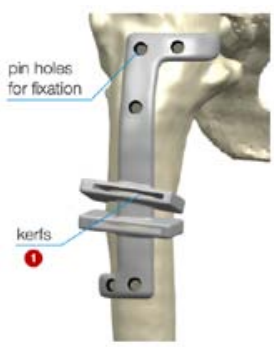

Figure $4 a$

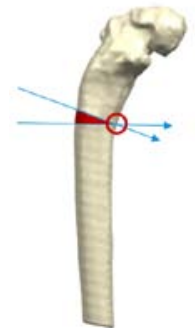

Figure $4 b$

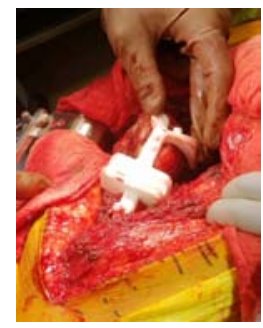

Figure $4 c$
Figure 4. Proposed design (a) for a custom-cutting jig with pin holes to secure the guide in its pre-planned location and saw blade guides oriented to obtain the desired angular correction. The saw guides were designed to accommodate a specific saw blade kerf to minimize toggle and maximize precision of the cuts at the projected angles (b). (c) An intraoperative photograph of the custom- cutting guide in position along the anterolateral aspect of the proximal femur. The guide was fabricated with medical-grade resin using 3-dimensional printing technology.

Planning the reconstructive portion of the case was done based on the predicted femoral model after the osteotomy (Fig. 3). An MP-Link modular stem (Waldemar Link GmbH and Co.; Hamburg, Germany), a Wagner-type stem, was chosen to bypass the abnormal proximal metaphysis and osteotomy site to obtain fixation in the distal, well-preserved femur. A modular stem (rather than a monoblock) was chosen in this case as we felt the ability to make height and version adjustments with a modular device was of added benefit compared to a one-piece stem. Together with the engineering team, implant specifications including optimal stem placement, size and version were determined preoperatively (Fig. 5). This advanced planning allows for what amounts to a virtual surgery.

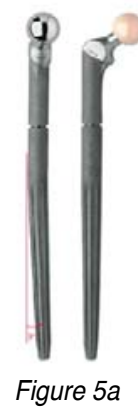

Figure $5 a$

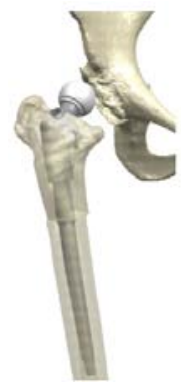

Figure $5 b$

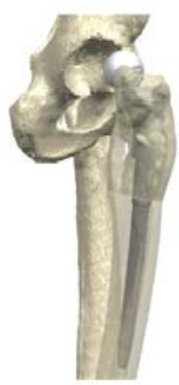

Figure $5 c$
Figure 5. (a) The proposed implant design included a stem with a 3-degree built-in angle to match that of the femur, as well as projected stem length, proximal and distal stem diameter, neck length and neck shaft angle. Anterior (a) and lateral (b) views showing the optimal position of the proposed implant within the femur after corrective alignment had been obtained.

The surgery was made difficult due to the prior surgical procedures, dense scar and contractures. Simple mobilization of the soft tissues, scar resection and tenotomies (psoas insertion and proximal adductors as well as the external rotators) allowed improved visualization. The custom cutting guide utilized for the osteotomy conformed to the patient's anatomy over the anterolateral femur in the preplanned site (Fig. 4). The custom jig was secured with smooth, trocar tipped $3.2 \mathrm{~mm}$ pins for fixation and the osteotomy was performed; after our osteotomy the bone ends were directly apposed which confirmed the adequacy of our cuts. The patient's cortical bone was extremely sclerotic and required the use of a high-speed drill to prepare the proximal canal. Standard reamers for the femoral implant were passed onto the distal segment beyond the osteotomy site. Once the final femoral implant was seated with bony apposition noted the osteotomy site, demineralized bone matrix putty was applied with a contoured plate and cable construct applied. The patient had an uneventful recovery and was allowed to fully weight bear and participate in physical therapy.

Postoperatively the patient had 2 out of 10 pain which went on to resolve completely. She had no residual groin or thigh pain. At 2 years postoperatively, the patient can walk unlimited distances and stair climb with an alternating gait. Her limb length inequality is significantly improved at 1.5 $\mathrm{cm}$ compared to $3 \mathrm{~cm}$ preoperatively. On exam she has 80 degrees of hip flexion, 20 degrees of abduction, 10 degrees 
adduction, 20 degrees of external rotation and 10 degrees of internal rotation. Her hip muscular strength is now graded at $5 / 5$.

Imaging 2-years postoperatively reveal a well fixed and aligned THA, though with persistent diastasis noted at the osteotomy site. It should be noted that at the time the intraoperative cuts were made, the bone edges were noted to be well-opposed, though the patient's bone in this area was sclerotic and noted to have poor bleeding quality. Postoperative radiographs demonstrated gradual resorption of bone around the osteotomy site, with the most recent radiographs showing persistent diastasis with incomplete bridging, though close inspection does find bridging bone across along the titanium grit blasted stem. We suspect that because of the poor bone quality noted in this area in conjunction with stress shielding from the diaphyseal-fitting stem, the patient may not ever develop quality bridging in this area (Fig. 6).
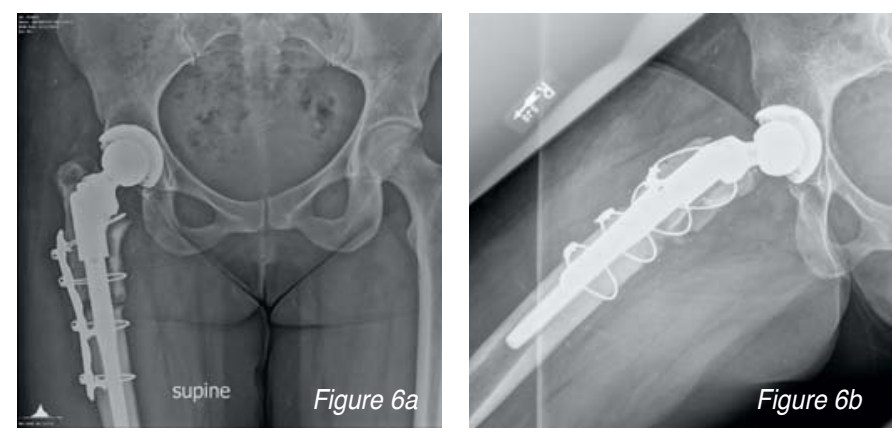

Figure 6. Radiographs showing an (a) anteroposterior view of the pelvis and $a(b)$ cross-table lateral view of the right hip obtained 2 years postoperatively. Imaging shows the implants remain in good position and good diaphyseal fit and bony on-growth distally without evidence of loosening. Osteotomy gap remains visible though scant bridging callous can be seen adjacent to the femoral stem.

\section{Discussion}

Proximal femoral deformities can lead to serious functional deficits and joint pain ultimately requiring treatment with end-prosthetic reconstruction. For cases with severe deformity, corrective osteotomy and THA are both needed. Corrective osteotomy can restore normal bone and soft tissue anatomy and re-establish mechanical alignment of the lower extremity. [14] THA can relieve pain and improve the limb function. [15]

3D-imaging software utilizes preoperative CT imaging to construct a $3 \mathrm{D}$ model which can be a valuable resource for virtual preoperative surgical planning as well as allow for preoperative collaboration with an engineering team for biomechanical validation of surgical plan. 3D surgical planning in conjunction with 3D printing technology has allowed for the development of custom surgical templates and choice of optimal implant specifics based on biomechanical evaluation. [16] The importance of a properly aligned corrective osteotomy in this case cannot be overstated for this young patient, as it will affect the longterm viability of her THA implants.

Choice of osteotomy size and position is key in providing optimal angular correction without creating a new deformity, and requires both thorough surgical planning and precise execution. [17] The optimal osteotomy should involve a minimal number of cuts and aim to re-establishing neck-shaft angle and restore the lower limb mechanical axis. [18] While classic freehand techniques have traditionally been used for corrective osteotomies, results tend to have larger deviations from the target correction as compared with patient-specific cutting guides (PSGs). PSGs assists in locating the correct osteotomy plane, as the guide fits uniquely into the correct position determined preoperatively. PSG for osteotomies have been associated with higher precision compared to freehand techniques, decreased radiation exposure, shorter operative times and decreased overall blood loss. [19-22]

In this case, a single lateral closing wedge osteotomy was planned in a position to address malalignment in both the coronal and sagittal planes. After osteotomy planning, custom cutting jigs were designed to fit the unusual deformity in this patient and allowed for accurate replication of our planned osteotomy and the desired degree of correction.

Use of long-stemmed femoral implants has been advocated in patients with PFD, particularly those with associated poor bone quality, in order to decrease the risk of aspetic loosening and early failure. [15] This can be challenging depending on the degree of deformity and the morphology of the medullary canal. 3D reconstructive imaging can be used to better characterize the sites of maximal deformity, the length of affected bone, overall bone quality, and determine canal diameter at multiple levels.

For this patient, preoperative 3D planning was used to determine our optimal component size and stem length given the predicted post-correction morphology of the femoral canal. Determining implant specifications preoperatively rather than intraoperatively decreases overall operative time and decreases the risk for cortical perforation or fracture during canal preparation. [15]

Despite the surgical advantages of 3D planning and printing patient specific instrumentation, the cost and time required for use should be considered. A 3D planning service and the production of custom resection guides as described above may cost between $\$ 4,000$ and $\$ 7,500$ de- 
pending on the complexity and number of required parts. The time spent on collaboration between surgeon and production team may take 4-5 weeks to fabricate the required instruments. [21] While the upfront costs are significant, use has been shown to offset some surgical costs related to operative time and fluoroscopy use, [를 as well as the potential savings provided by avoiding complications and revision surgery. Implant costs for revision of the femoral component alone may cost nearly $\$ 10,000$, with overall costs to the hospital being more than double that. $[\underline{24}, \underline{25}]$

\section{Summary}

3D design software for THA is a valuable resource for planning proximal femoral corrective osteotomies as well as determining implant size and design specifications in patients with proximal femoral deformities. 3D printing patient-specific cutting guides can help improve the accuracy of osteotomy cuts to reproduce the same degree of correction outlined in the surgical plan. The additional cost and time investment for these resources is worthwhile in patients with severe deformity and high surgical complexity.

\section{References}

1. S. M. J. Mortazavi, C. Restrepo, P. J. W. Kim, J. Parvizi, and W. J. Hozack, "Cementless femoral reconstruction in patients with proximal femoral deformity," J. Arthroplasty, vol. 26, no. 3, pp. 354-359, Apr. 2011, doi: 10.1016/j. arth.2010.09.002.

2. X. Deng et al., "Total hip arthroplasty with femoral osteotomy and modular prosthesis for proximal femoral deformity," J. Orthop. Surg., vol. 14, no. 1, p. 282, Aug. 2019, doi: 10.1186/s13018-019-1336-1.

3. L. Helgesson, P. K. Johansson, Y. Aurell, C.-J. Tiderius, J. Kärrholm, and J. Riad, "Early osteoarthritis after slipped capital femoral epiphysis," Acta Orthop., vol. 89, no. 2, pp. 222-228, Apr. 2018, doi: 10.1080/17453674.2017.1407055.

4. F. T. Hoaglund and L. S. Steinbach, "Primary osteoarthritis of the hip: etiology and epidemiology," J. Am. Acad. Orthop. Surg., vol. 9, no. 5, pp. 320-327, Oct. 2001, doi: 10.5435/00124635-200109000-00005.

5. V. Khanna and P. E. Beaulé, "Defining structural abnormalities of the hip joint at risk of degeneration,” J. Hip Preserv. Surg., vol. 1, no. 1, pp. 12-20, Jul. 2014, doi: 10.1093/jhps/hnu004.

6. D. A. Goodman, J. E. Feighan, A. D. Smith, B. Latimer, R. L. Buly, and D. R. Cooperman, "Subclinical slipped capital femoral epiphysis. Relationship to osteoarthrosis of the hip," J. Bone Joint Surg. Am., vol. 79, no. 10, pp. 1489-1497, Oct. 1997, doi: 10.2106/00004623-199710000-00005.

7. T. M. Ecker, M. Tannast, M. Puls, K. A. Siebenrock, and S. B. Murphy, "Pathomorphologic alterations predict presence or absence of hip osteoarthrosis," Clin. Orthop., vol. 465, pp. 46-52, Dec. 2007, doi: 10.1097/BLO.0b013e318159a998.

8. L. Al-Mouazzen, K. Rajakulendran, and N. Ahad, "Fibrous dysplasia, shepherd's crook deformity and an intra-capsular femoral neck fracture," Strateg. Trauma Limb Reconstr., vol. 8, no. 3, pp. 187-191, Nov. 2013, doi: 10.1007/s11751-0130174-7.

9. Y. Hagiwara, S. Iwata, T. Yonemoto, and T. Ishii, "Rotational valgus osteotomy for shepherd's crook deformity: a case report,” J. Orthop. Sci. Off. J. Jpn. Orthop. Assoc., vol. 20, no. 2, pp. 422-424, Mar. 2015, doi: 10.1007/s00776-013-0463-5.

10. F. Hefti, L. Donnan, and A. H. Krieg, "Treatment of shepherd's crook deformity in patients with polyostotic fibrous dysplasia using a new type of custom made retrograde intramedullary nail: a technical note," J. Child. Orthop., vol. 11, no. 1, pp. 64-70, 2017, doi: 10.1302/1863-2548.11.170002.

11. K. Watanabe, H. Tsuchiya, K. Sakurakichi, H. Matsubara, and K. Tomita, "Dou- ble-level correction with the Taylor Spatial Frame for shepherd's crook deformity in fibrous dysplasia," J. Orthop. Sci. Off. J. Jpn. Orthop. Assoc., vol. 12, no. 4, pp. 390-394, Jul. 2007, doi: 10.1007/s00776-007-1132-3.

12. K. Sakurakichi, H. Tsuchiya, T. Yamashiro, K. Watanabe, H. Matsubara, and K. Tomita, "Ilizarov technique for correction of the Shepherd's crook deformity: a report of two cases," J. Orthop. Surg. Hong Kong, vol. 16, no. 2, pp. 254-256, Aug. 2008, doi: 10.1177/230949900801600226.

13. X. Zhang, C.-Y. Chen, H. Duan, and C. Tu, "Multiple valgus osteotomies combined with intramedullary nail for shepherd 's crook deformity in polyostotic fibrous dysplasia: a case series and review of the literature," 2016. I paper/Multiple-valgus-osteotomies-combined-with-nail-for-Zhang-Chen/bc8f7118820652c4b15b30e7947bf27768f226d5 (accessed Nov. 24, 2020).

14. M. R. DiCaprio and W. F. Enneking, "Fibrous dysplasia. Pathophysiology, evaluation, and treatment," J. Bone Joint Surg. Am., vol. 87, no. 8, pp. 1848-1864, Aug. 2005, doi: $10.2106 / J B J S . D .02942$.

15. R. J. Sierra and M. E. Cabanela, "Total hip arthroplasty in patients with underlying fibrous dysplasia," Orthopedics, vol. 32, no. 5, p. 320, May 2009, doi: 10.3928/01477447-20090501-14.

16. K. C. Wong, S. M. Kumta, N. V. Geel, and J. Demol, "One-step reconstruction with a 3D-printed, biomechanically evaluated custom implant after complex pelvic tumor resection," Comput. Aided Surg. Off. J. Int. Soc. Comput. Aided Surg., vol. 20, no. 1, pp. 14-23, 2015, doi: 10.3109/10929088.2015.1076039.

17. M. Jacobi, P. Wahl, S. Bouaicha, R. P. Jakob, and E. Gautier, "Distal femoral varus osteotomy: problems associated with the lateral open-wedge technique," Arch. Orthop. Trauma Surg., vol. 131, no. 6, pp. 725-728, Jun. 2011, doi: 10.1007/s00402010-1193-1.

18. J. G. Boldt, J.-C. Cartillier, A. Machenaud, and J.-P. Vidalain, "Long-term Bone Remodeling in HA-coated Stems: A Radiographic Review of 208 Total Hip Arthroplasties (THAs) with 15 to 20 Years Follow-up," Surg. Technol. Int., vol. 27, pp. 279-286, Nov. 2015.

19. L. O. A. Thanni and N. O. Aigoro, "Surgical site infection complicating internal fixation of fractures: incidence and risk factors," J. Natl. Med. Assoc., vol. 96, no. 8, pp. 1070-1072, Aug. 2004.

20. J. Moore, L. Mychaltchouk, and F. Lavoie, "Applicability of a modified angular correction measurement method for open-wedge high tibial osteotomy," Knee Surg. Sports Traumatol. Arthrosc. Off. J. ESSKA, vol. 25, no. 3, pp. 846-852, Mar. 2017, doi: 10.1007/s00167-015-3954-4.

21. G. Sys, H. Eykens, G. Lenaerts, F. Shumelinsky, C. Robbrecht, and B. Poffyn, "Accuracy assessment of surgical planning and three-dimensional-printed patientspecific guides for orthopaedic osteotomies," Proc. Inst. Mech. Eng. [H], vol. 231, no. 6, pp. 499-508, Jun. 2017, doi: 10.1177/0954411917702177.

22. P. Zheng, P. Xu, Q. Yao, K. Tang, and Y. Lou, "3D-printed navigation template in proximal femoral osteotomy for older children with developmental dysplasia of the hip," Sci. Rep., vol. 7, p. 44993, 21 2017, doi: 10.1038/srep44993.

23. D. H. Ballard, P. Mills, R. Duszak, J. A. Weisman, F. J. Rybicki, and P. K. Woodard, "Medical 3D Printing Cost-Savings in Orthopedic and Maxillofacial Surgery: Cost Analysis of Operating Room Time Saved with 3D Printed Anatomic Models and Surgical Guides," Acad. Radiol., vol. 27, no. 8, pp. 1103-1113, Aug. 2020, doi: 10.1016/j.acra.2019.08.011.

24. C. U. Gwam et al., "Current Epidemiology of Revision Total Hip Arthroplasty in the United States: National Inpatient Sample 2009 to 2013,” J. Arthroplasty, vol. 32, no. 7, pp. 2088-2092, Jul. 2017, doi: 10.1016/j.arth.2017.02.046.

25. K. D. Collins, K. K. Chen, J. D. Ziegler, R. Schwarzkopf, J. A. Bosco, and R. Iorio, "Revision Total Hip Arthroplasty-Reducing Hospital Cost Through Fixed Implant Pricing," J. Arthroplasty, vol. 32, no. 9S, pp. S141-S143, Sep. 2017, doi: 10.1016/j.arth.2017.02.082. 
SUBMISSION HISTORY

Submitted: March 4, 2021

Reviewed: July 21, 2021

Accepted: September 15, 2021

Published: October 12, 2021

AUTHOR AFFILIATIONS

1 Zachary C. Hanson, MD; Donald D. Davis, MD; J. Weston Robison, MD Wellstar Atlanta Medical Center, 303 Parkway Dr NE, Atlanta, GA

2 Jon E. Minter, DO

Orthopaedic Surgeon, Northside Total Joint Specialists, Alpharetta , GA

(Direct inquires to Zachary Hanson, zchg47@gmail.com)
AUTHOR DISCLOSURES

- The authors declare that there is no conflict of interest in connection with this submitted article.

COPYRIGHT \& OPEN ACCESS

(C) 2021 Hanson, Davis, Robison, Minter. All rights reserved. Authors retain copyright and grant the journal right of first publication with the work. Reconstructive Review is an open access publication and

follows the Creative Commons Attribution-NonCommercial CC BY-

NC. This license allows anyone to download works, build upon open 6 access the material, and share them with others for non-commercial purposes as long as they credit the senior author, Reconstructive Review, and the Joint Implant Surgery \& Research Foundation (JISRF). An example credit would be: "Courtesy of (senior author's name), Reconstructive Review, JISRF, Chagrin Falls, Ohio". 